\title{
X8.2 Solar Flare on The Rear Side of the Solar Disk: An Evidence for the Current Sheet as a Mechanism for Cosmic Ray Acceleration
}

\author{
I.M. Podgorny ${ }^{1}$, A.I. Podgorny ${ }^{2}$ \\ ${ }^{1}$ Institute of Astronomy RAS, Moscow, Russia \\ ${ }^{2}$ Lebedev Physical Institute RAS, Moscow, Russia \\ E mail (podgorny@inasan.ru).
}

Accepted: 14 January 2019

\begin{abstract}
The X8.2 flare occurred on 2017 September 11 during the minimum period of solar activity. The flare is appeared over the active region AR12673. This active region produces two large and many small flares. The solar cosmic rays have been observed in association with the X8.2 flare. The active region at that time is situated on the back side of the Sun disk behind the Western limb. The front of the accelerated protons flux arrives to the Earth with the delay not exceeding the proton flight time from the Sun. Such proton propagation can occur only along the lines of the interplanetary magnetic field. The solar proton acceleration takes place by electric filed along a singular line of the current sheet above an active region. There is no reason to believe that the mechanisms of cosmic ray acceleration on the Sun and other stars are of different nature. The results of observations of accelerated electrons on the SOHO/EPHIN instrument show that electrons are accelerated and propagated during flares together with protons. At the same time, a simple analysis of the GOES results leads to the conclusion that the flux of relativistic electrons does not show any connection with the recorded solar cosmic rays. Currently, this contradiction cannot be explained. Photos in spectral lines of ions obtained on the SDO spacecraft are used to study the pre-flare state and flare development. The source of flare radiation in the spectral lines of the highly ionized irons FeXXIV and FeXXIII is observed in the corona outside the solar limb. The emission of these spectral lines sharply increases during appearing of flare X-ray radiation. The energy release of a flare occurs in the corona above an active region. The temperature in the flare is greater than $20 \mathrm{MK}$. The size of the hot plasma cloud is $\sim 10^{10} \mathrm{~cm}$.
\end{abstract}

\section{(c) 2019 BBSCS RN SWS. All rights reserved}

Keywords: solar corona, solar flare, current sheet, cosmic rays, spectral lines of highly ionized ions

\section{Introduction $^{1}$}

The solar constant indicates an amazing stability of the solar thermonuclear reactor. According to extra-atmospheric measurements, the solar constant is $1367 \mathrm{~W} / \mathrm{m}^{2}$. The solar constant is the total flux of solar radiation passing per unit time through a single area, oriented perpendicular to the radiation flow, at a distance of one astronomical unit from the Sun outside the terrestrial atmosphere. The variation of the solar constant during the 11-year cycle of solar activity, apparently, does not exceed $\sim 10^{-3}$. Against the backdrop of this amazing stability of the Sun's work the big solar flares are observed several times per a year. The observed flare energy release takes place in the corona over a complex active region with a magnetic flux greater than $10^{22} \mathrm{Mx}$. Solar flares usually occur during periods of the high solar activity. The energy of a solar flare can exceed $10^{32} \mathrm{erg}$. The flare duration is from 10 to 100 minutes. The flare is a manifestation of a number of physical processes, including X-ray radiation, the ejection of the corona substance, and proton acceleration to the relativistic energy of not less than $20 \mathrm{GeV}$ (Balabin, et al. 2005; Podgorny, Podgorny, 2016).

The Sun is the only astronomical object that generates observed in the solar system proton pulses with the relativistic energy. The solar proton acceleration takes place along a singular line of the current sheet above an active region. The electric field $\mathbf{E}=-\mathbf{V} \times \mathbf{B} / \mathbf{c}$ for proton acceleration in a flare current sheet is generated at magnetic lines reconnection. The accelerated proton spectra coincide with the spectra calculated for acceleration in the current sheet that simulated numerically by MHD models of real solar flares (Balabin, et al. 2005; Podgorny, Podgorny, 2016). The pulses of relativistic protons accompanying flares are recorded against the background of a continuous flux of galactic cosmic rays with the energy of more than $10^{15} \mathrm{eV}$. Their acceleration occurs outside the solar system. For more than 100 years the galactic cosmic ray acceleration has been studied, but the physical mechanisms of acceleration remain unclear. The most popular, but unproven hypothesis, is the galactic proton acceleration in interstellar shock waves. It is impossible to exclude that galactic cosmic rays are accelerated by the same mechanism as the solar cosmic rays. However, the energy of galactic cosmic ray particles is several orders of magnitude higher than the maximum energy of protons accelerated on the Sun. This fact for a long time does not allow us to state unambiguously that the acceleration of galactic and solar cosmic rays may occur by the same mechanism. The modern observation of giant flares on the star dwarfs of the class G (Maehara, et al. 2012; Shibayama. et al. 2013) with the energy significantly exceeding the energy of the solar flares indicates the possibility of proton acceleration beyond the boundary of the solar system to energies significantly greater than the energy of solar cosmic rays. The generation of superflares with the energy much greater than the energy of big solar flares on variety of class $G$ stars is reported, some of which are rapidly rotating and some of which are of an ordinary solar type (Maehara, et al. 2012). 365 superflares are observed on the stars, including some superflares that are generated on the slowly rotating solar-type stars. About 83,000 stars have been investigated over 120 days using Kepler spacecraft data (Maehara, et al. 2012).

The previously considered significant difference in the energy maximums of galactic and solar cosmic rays did not contribute to the idea of the same cosmic ray acceleration mechanisms on the Sun and on stars. The recent data (Shibayama, et al., 2013) are showed that the energy of the stellar flare can exceed $10^{36} \mathrm{erg}$. It is by 3-4 orders greater than the energy of a large solar flare, and, apparently, the energy of the protons accelerated in these stellar flares can significantly exceed the energy of the particles registered from flares on the Sun. The flare and dynamics of the pre-flare state of the active region that caused the flare are available for direct investigation only on the Sun. Such possibility is not existed for stars. 


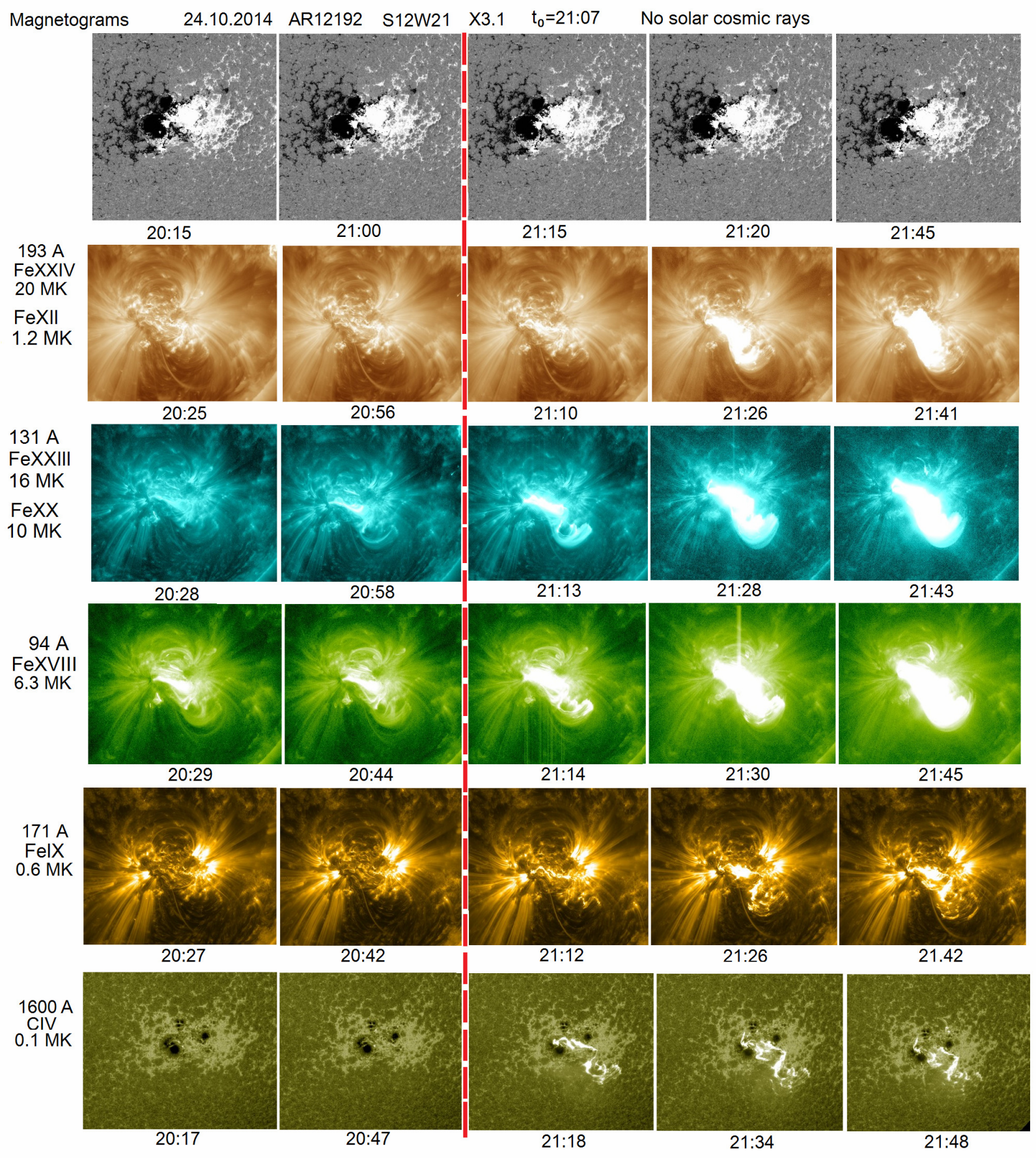

Fig. 1. Flare emissions in the pre-flare state and during the flare occurrence state observed in the middle of the solar disk in spectral lines of atoms of different degrees of ionization.

The solar flare physics is very important branch of the modern astronomy. The solar flare is a complex physical phenomenon with the energy release above $10^{32} \mathrm{erg}$, which causes perturbation of the Earth's magnetic field and often leads to unpleasant economic consequences. Solar cosmic rays with the energy of at least $20 \mathrm{GeV}$, often accompanying a solar flare, produce a serious threat to the health of crews in planned interplanetary missions. The prediction of solar flare appearance is the most important task of the modern solar physics.

\section{The flare in spectral lines of highly ionized iron}

The flare development is demonstrated in the spectral lines of highly ionized atoms (Podgorny I.M., Podgorny A.I. 2017, 2018) detected with the Atmospheric Imaging Assembly instrument on the Solar Dynamic Observatory (SDO AIA) (https://sdo.gsfc.nasa.gov/data). A typical development of a solar

flare produced by the active region that situated near the solar disk center is shown in Fig. 1. To the left of the vertical (red) line the frames are shown that taken before the flare. The flare X3.1 appears above an active region on September 24, 2014 at $\mathrm{t}_{0}=21$ : 07 . The photos presented to the right of the vertical red line show the flare development.

The first line of Fig. 1 shows one of the most fundamental properties of a solar flare - the practical constant of the magnetic field sources on the photosphere during hours immediately before the flare and during the flare. The results of the numerical MHD simulation of the flare shows (Podgorny A.I., Podgorny I.M. 2012; 2013) a very slow (for several days) formation of the preflare magnetic configuration (current sheet) in the corona over an active region. At that time the current system of a current sheet is created in the corona above an active region. Very slow dissipation of the magnetic field of this current sheet takes place in this time. The flare appears at $t_{0}=21: 07$. Noticeable perturbations 

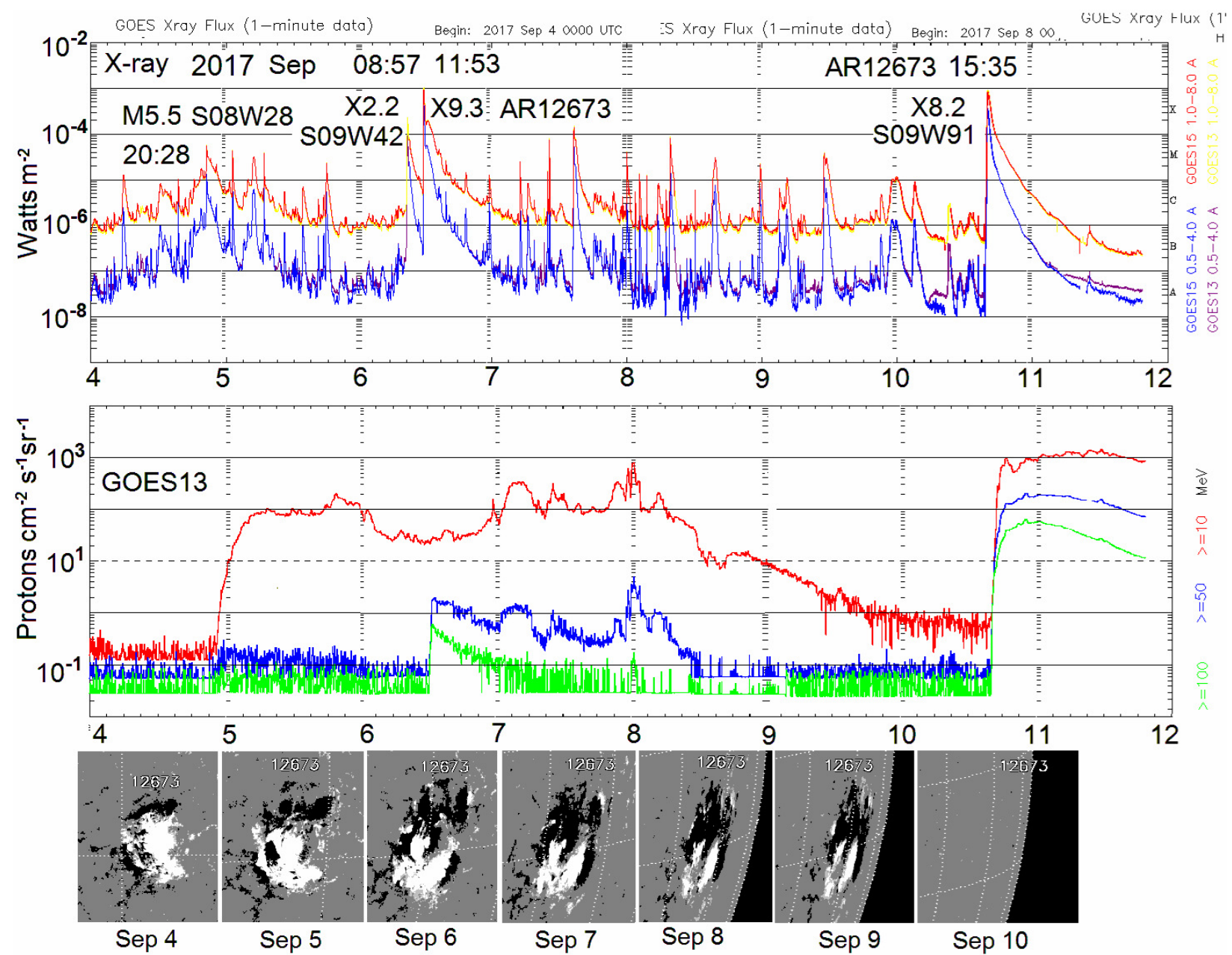

Fig. 2. The event 4 - 10 September, 2017 at the solar activity minimum and the magnetic field evolution in the active region AR12673. The magnetograms are shown.

of the magnetic field on the photosphere during the flare are not observed. In numerical MHD simulation (Podgorny A.I., Podgorny I.M. 2012; 2013), the initial and boundary conditions on the photosphere are determined from measurements of the preflare state of the active region for several days before a real flare. The simulation results show that a current sheet in the corona is formed before the flare. The magnetic field energy that accumulated in this current sheet is realized during a flare.

The second line of Fig. 1 shows a very slight increase in the emission of the hot plasma spectral line of $193 \AA$ FeXXIV before the flare, but this emission sharply increases during the flare. The measuring channel of the 193A spectral line on the SDO AIA can record the emission of spectral lines of iron ions FeXXIV $\left(\mathrm{T}_{\mathrm{e}}=20\right.$ $\mathrm{MK})$ and FeXII $\left(\mathrm{T}_{\mathrm{e}}=1.2 \mathrm{MK}\right)$. When a big flare occurs and develops, thermal $\mathrm{X}$-ray radiation indicates the plasma temperature $40 \mathrm{MK}$ (LIN ET AL., 2003). At such temperature, the FeXII ion should be completely burned out, and its emission during a flare cannot contribute to the 193 A line emission. Emission and dimming radiation of the ion FeXII can take place only at slightly heated corona.

The third line (131 ̊ FeXXIII line) also demonstrates the beginning of slight heating of the coronal plasma cloud during the current sheet formation above the active region and the rapid heating of this plasma during a flare. Photos in the fourth line indicate preheating of the coronal plasma cloud during the formation of the current sheet and the development of the emission of the $94 \AA$ FeXVIII line in the flare. The fifth and sixth lines demonstrate small torn chromospheric structures - flare filaments, which for a long time have been considered as the main flare manifestation.

Not an ordinary event is shown in Fig. 2. In solar activity minimum, when powerful active regions are rarely observed, the developing of the active region is appeared on the eastern limb. When the active region is moving along the disk, its development is accompanied by a series of solar flares of various sizes. The first flare of M5.5, which appeared near the center of the Sun, (S08W28) develops unusual. The front of its X-ray radiation is tightened by two orders of magnitude in comparison with the normally recorded flares.

The last flare X8.2 on September 10, 2017 at 15:35 is appeared above the active region that situated on the solar disk back side. The flare active region is not seen on the disk during the flare (Fig. 2). It situated on the solar back side, but the hot spectral line emissions (193 $\AA$ and $131 \AA$ ) in Fig. 3 demonstrate plasma cloud heating in the corona during the flare. Fig. 3 shows that emission of the hot line $94 \AA$ (6.3 MK) is appeared before the flare X8.3 at current sheet creation in the corona. Emission of the $94 \AA$ line is increased sharply during the flare, but as for strong very hot lines $193 \AA(20 \mathrm{MK})$ and $131 \AA$ (16 MK) they appear only during the flare. These photos can be considered as a new independent evidence of the flare appearing in the corona, but not in the chromosphere. The flare emissions occur in the corona outside the solar disk, as it should be in the case, if the energy is realized in the corona. This energy has been stored in the magnetic field of a current sheet in the corona above the active region. 


\subsubsection{X8.2 S09W91 AR12673 $t_{0}=15: 25$}

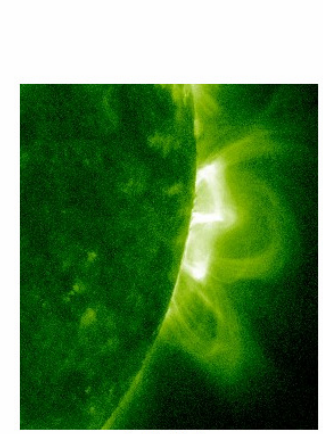

$14: 30$

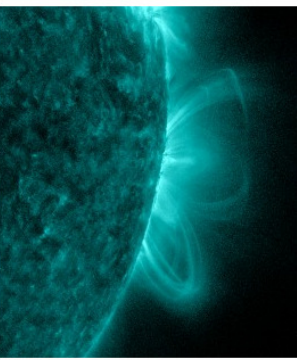

$14: 27$

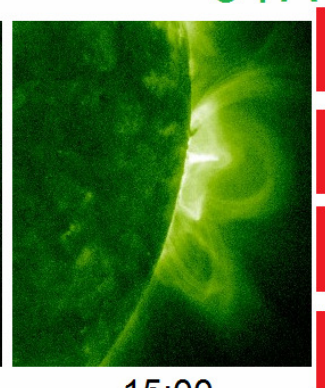

15:00

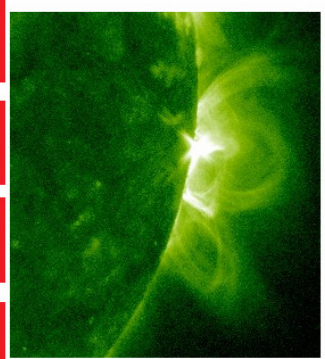

$15: 45$

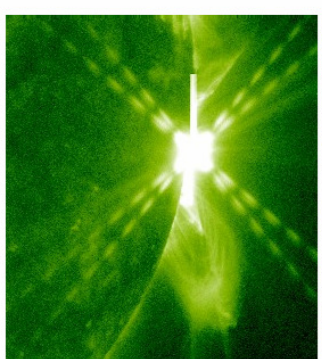

16:00

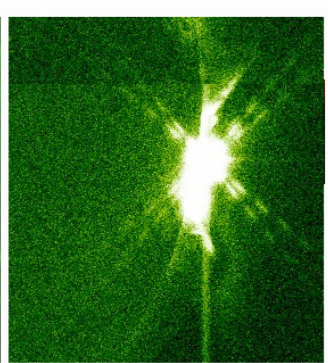

$16: 14$

131 A FeXXIII 16 MK, FeXX 10 MK

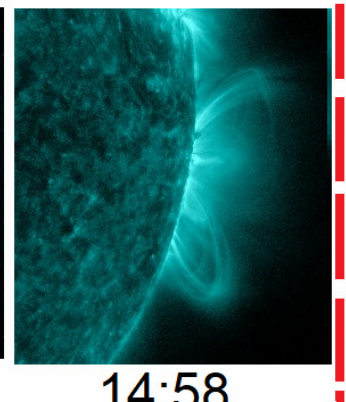

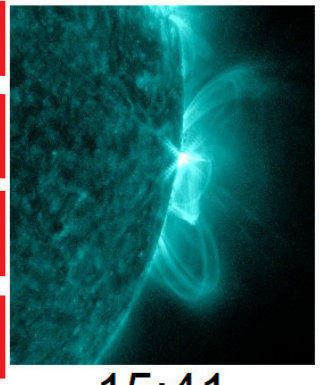

15:41

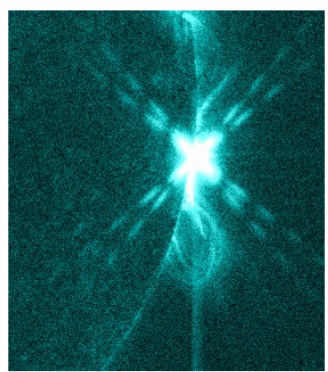

$15: 58$

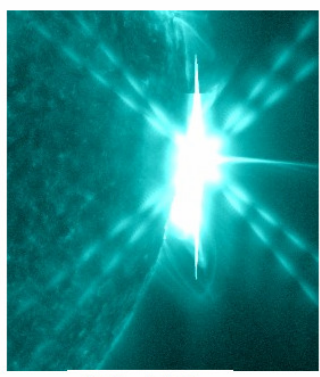

$16: 28$

193 A FeXXIV 20 MK

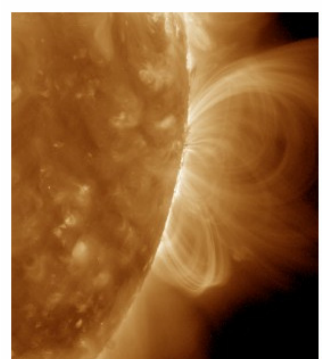

$14: 26$

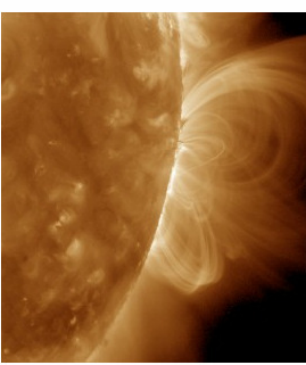

$14: 56$

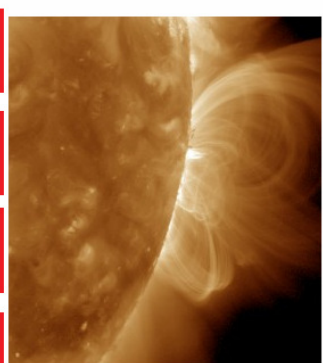

$15: 41$

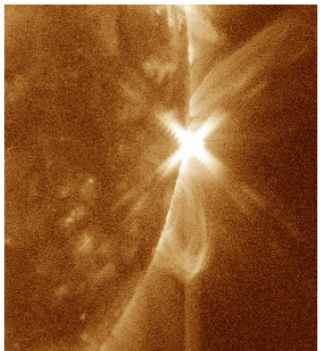

$15: 56$

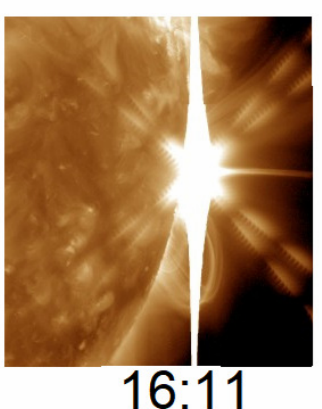

Fig. 3. The flare X8.2 in the hot spectral lines $94 \AA, 131 \AA$ and $193 \AA$. The active region is located behind the visible side of Sun. The heating of a plasma cloud during the flare occurs in the corona beyond the solar disk boundary.

\section{Solar cosmic rays}

In the September 2017 high energy protons are arrived to the Earth from several large flares (Fig. 2). Flares smaller than $\mathrm{M}$ type do not create a flux of accelerated protons. Fluxes of protons from different flares are superimposed on each other, and it is impossible to analyze the proton arrival delay for the most flares. The exception is the flare X8.2, which occurs over the active area on the back side of the Sun. The delay of the front of the arrival protons relative to the start of the flare does not exceed an hour, and the width of the front flux is about $\sim 30 \mathrm{~min}$. This means that the proton flux front from the flare on the back side of the Sun propagates with the velocity of accelerated protons. Such propagation of protons can occur from a flare only in the absence of collisions, and the delay is determined by the transit time of the particle moving along the line of the interplanetary magnetic field, which has the form of an Archimedes spiral.

Fig. 4 shows the proton fluxes that are accelerated in different regions of the solar disk and are recorded near the Earth. Protons from flare accelerated in the Western part of the solar dick can arrive to the Earth along interplanetary magnetic field lines with their velocity without collisions. For comparison the typical proton fluxes from flares that occurred near the center of the solar disk and near the eastern limb are also presented. These protons can propagate to the Earth due to diffusion across the magnetic field. They demonstrate a very soft front, which arrives with the delay of several hours. Fig. 4 shows that the protons, which occurred Sun near the western limb, arrive to the Earth with a sharp front $\sim 30 \mathrm{~min}$ and a delay which equals to the time of flight of the particle between the flare and the spacecraft. Such a process can occur when particles are moving on the flux front without collisions along the magnetic field line of Archimedes' spiral. The arrival of the front of the particles from flares that appeared in the center of the disk (N15E02) and near the east limb (S15E65) occurs only with a much longer delay, not less than $\sim 3$ hours. Particles from such flares can arrive to Earth only across the lines of the magnetic field. The front of the proton flux from these flares is a rather flat with duration of 10 to 20 hours. The delay in the arrival of the proton flux front from the flares of the eastern and central parts of the disk is clearly less than the time of particle transfer by the solar wind. Apparently, their traveling time is determined by the diffusion of protons across the magnetic field due to scattering by field fluctuations caused by beam instability (Istomin, 2010). 

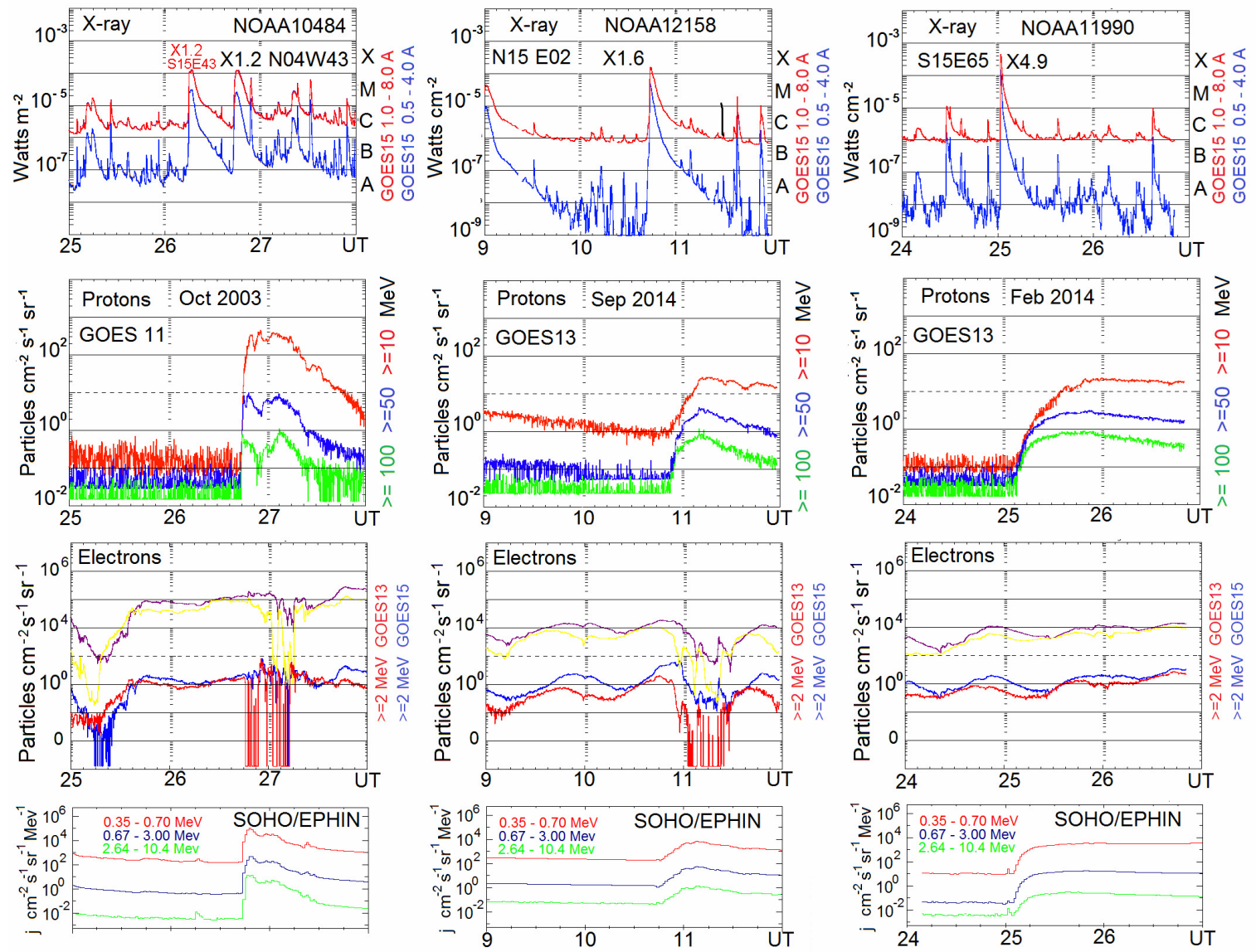

Fig. 4. The typical solar cosmic ray fluxes, recorded in the Earth orbit, from flares that occurred in various regions of the solar disk. Left, central, and right figures columns shows solar cosmic ray fluxes from region near the western limb, near the center of the solar disk, and near the eastern limb.

The flux of relativistic electrons is also recorded on the GOES spacecraft and by the SOHO/EPHIN instrument (http://www2.physik.uni-kiel.de/SOHO/phpeph/EPHIN.htm) (Fig. 4, 5). The flux of relativistic electrons measured with the SOHO/EPHIN instrument, which is situated out of the magnetosphere, show good connection with the accelerated protons flux. The behavior of relativistic electrons measured on the GOES spacecraft does not show any connection with the recorded solar cosmic rays. The GOES spacecraft is situated in upper part of magnetosphere (on the orbit with the radius $\sim 7 \mathrm{R}_{\mathrm{E}}$ whereas the dayside boundary of magnetosphere is on the distance $\sim 15 \mathrm{R}_{\mathrm{E}}$ from Earth center, where $\mathrm{R}_{\mathrm{E}}=6371 \mathrm{~km}$ is the Earth radius). At the first sight of view, the SOHO/EPHIN instrument measures the electrons accelerated in the flares, which are propagated in the interplanetary space, and field of magnetosphere do not give possibility for these fast electrons reach the GOES spacecraft. But simple analysis of possibility for fast electrons reach the GOES spacecraft basing on values of Larmor radiuses causes the questions about conclusions which can made from results of GOES measurements.

The Larmor radius of proton with energy $10 \mathrm{MeV}$ in magnetic field $100-150 \mathrm{nT}$ measured by GOES is $3054-4581 \mathrm{~km}$ $\left(\sim 0.5-0.7 \mathrm{R}_{\mathrm{E}}\right)$. The GOES measurements of protons with such energies are explained good by solar cosmic rays' propagation in the interplanetary space from the flares sites in the West and East parts, and behavior of proton flux detected on GOES coincides with proton measurements by SOHO/EPHIN which is situated out of magnetosphere. It means that field of magnetosphere do not interfere accelerated proton from interplanetary space reach the GOES spacecraft in spite of its Larmor radius near the GOES spacecraft is much less (in 10 - 14 times) then distance from spacecraft GOES to the dayside magnetosphere boundary. It can be seeming that $200 \mathrm{MeV}$ electrons cannot reach the GOES spacecraft because their Larmor radius near the GOES spacecraft equal to $54.65-81.97 \mathrm{~km}\left(\sim 10^{-2}-1.4 \times 10^{-2} \mathrm{R}_{\mathrm{E}}\right.$ in $500-700$ times less then distance from spacecraft GOES to the dayside magnetosphere boundary) is much less $10 \mathrm{MeV}$ proton Larmor radius near the GOES spacecraft. But $1 \mathrm{GeV}$ protons passed the magnetosphere to Earth atmosphere and registered by neutron monitors (see, for example, Balabin, et al. 2005). The Lamor radius of $1 \mathrm{GeV}$ proton near the Earth atmosphere (where the magnetic field is $1-0.7 \mathrm{G}$ ) equal to $56.56-80.79 \mathrm{~km}$ is the same as the Larmor radius of $200 \mathrm{MeV}$ electron near GOES spacecraft. The protons from the western flares, which arrive to the Earth moving along the magnetic field line of Archimedes' spiral, have anisotropic pitch-angle distribution in the interplanetary magnetic field near the boundary of the magnetosphere. They are measured only by several neutron monitors of worldwide network. The scattered protons which are registered during about three days after flares, occurred in western and eastern parts of solar disk, have isotropic pitch-angle distribution. They are measured by large number of neutron monitors of worldwide network. A neutron monitor located at some given point measures the flux of these scattered protons from a significant number of flares. If the behavior of electrons accelerated in the flares and propagated to the Earth is analogical to behavior of accelerated protons (as it can be concluded from data of SOHO/EPHIN instrument), then GOES have to detect from rather large number of flares scattered 200 $\mathrm{MeV}$ electrons, which have the same Larmor radius near the GOES spacecraft as $1 \mathrm{GeV}$ proton near the Earth atmosphere. But GOES measurements do not show increase of $200 \mathrm{MeV}$ electron flux during flares (see Fig 4, 5 for some flares). So GOES measurements of $200 \mathrm{MeV}$ electrons indicate, that during flares accelerated electrons can be absent in the interplanetary space. 

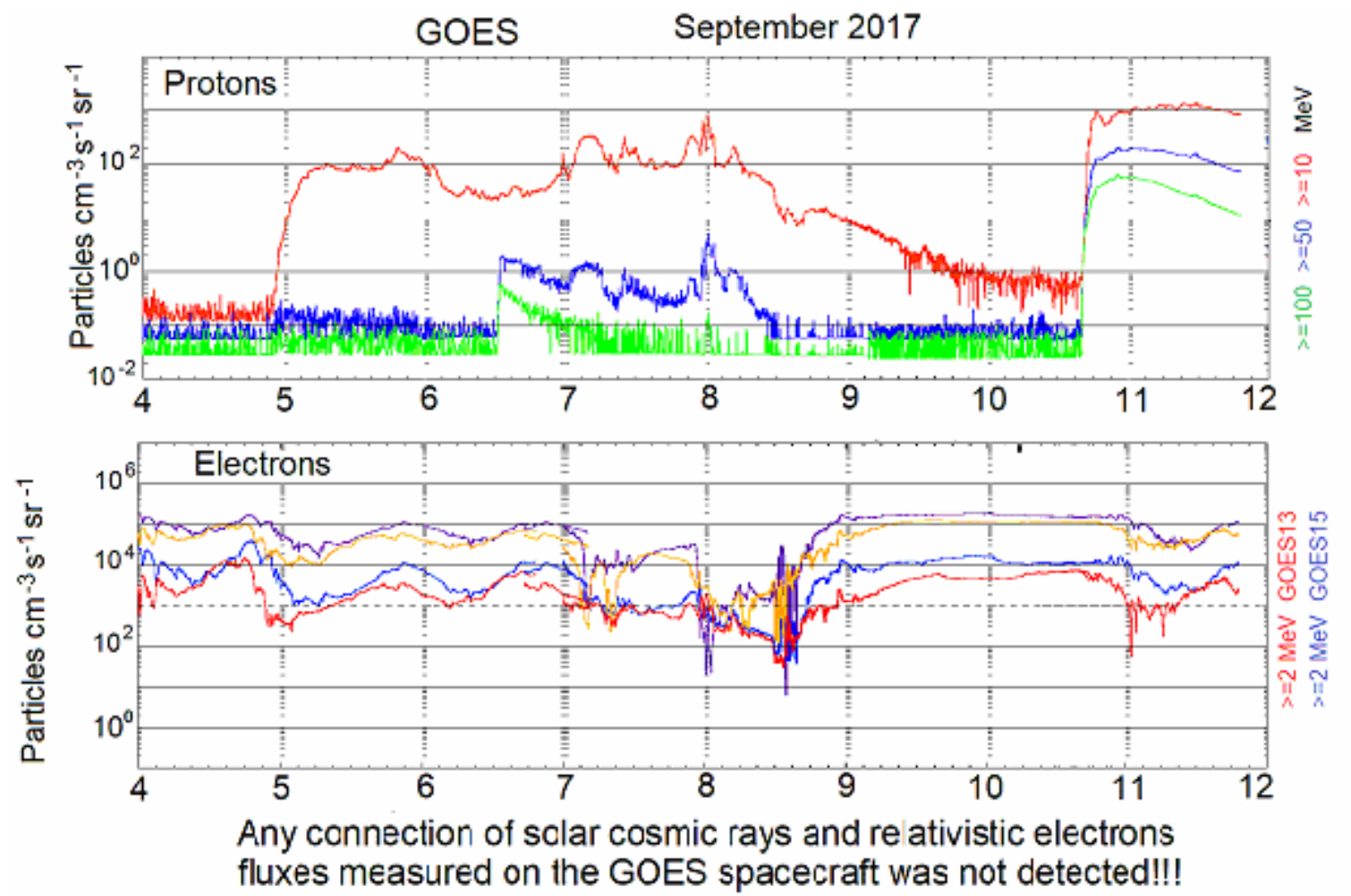

Fig. 5. The fluxes of accelerated protons near the Earth's orbit, generated by a series of flares, and fluxes of relativistic electrons.

Similar behavior of relativistic electrons, measured by SOHO/EPHIN, and protons indicates the possibility of the generation of electrons during a flare and their propagation together with protons in the interplanetary space. However, the analysis of GOES data indicates the absence of accelerated electrons during flares for reasons that have not yet been clarified.

\section{Cosmic ray acceleration on the Sun and in the galaxy}

The flare observation and MHD numerical simulation show that solar cosmic rays generation takes place in a flare current sheet. The pulse of fast protons is generated simultaneously with appearing of the flare. The measured spectra of solar cosmic rays coincide with the spectra calculated for acceleration in a flare current sheet. The exact spectra coincidence is observed at the reconnection rate $10^{7} \mathrm{~cm} / \mathrm{s}$. The measured spectra of solar cosmic rays are calculated for acceleration in a real flare current sheet (Podgorny, Podgorny, 2016), using observed initial and boundary conditions. The Sun is the only astronomical object that generates proton pulses with the energy of at least $20 \mathrm{GeV}$, which origin is accessible to direct research. Galactic cosmic rays have been discovered 100 years ago, but still there are no information about the mechanism of their acceleration. The most popular, but unproven hypothesis is the proton acceleration in the interstellar shock waves.

Thus, there is every reason to assert that solar cosmic rays and galactic cosmic rays are generated by the same mechanism. The temptation arises to declare that the same mechanism of galactic cosmic ray creation is developing on many stars. However, the energy of galactic cosmic rays is several orders of magnitude higher than the maximum energy of protons accelerated on the Sun. This fact for a long time did not allow us to state unambiguously that the acceleration of galactic and that solar cosmic rays occurs by the same mechanism.

The discovery of giant flares on the star dwarfs of the class $G$ (Maehara, et al. 2012; Shibayama. et al. 2013) with the energy significantly exceeding the energy of the solar flares brings new information. It is impossible to exclude that such gigantic flares can accelerate ions up to the energy much bigger than the energy of solar cosmic rays. The results of (Maehara, et al. 2012; Shibayama. et al. 2013) can be considered as a new independent argument in the favor of cosmic ray generating in flares, but not in shock waves. The flare is the universal astronomical process that responsible for proton acceleration on the Sun and on the stars. The flare and dynamics of the pre-flare state of the active region that caused the flare are available for direct observation only on the Sun. The solar flare is a complex physical phenomenon, which produces perturbation of the Earth's magnetic field and often leads to unpleasant economic consequences.

\section{Conclusion}

The flare is a universal astronomical process that generates cosmic rays. Flares occur on the Sun and stars. A detailed study of the mechanism of the flare is possible only on the Sun. The active region AR12673 has appeared at the solar activity minimum, when no flares are usually observed during a year. The release of the flare energy is occurred in the corona over the active region. The AR12673 region has produced series of large flares, including the X8.2 flare on the back side of the solar disk. The plasma cloud in the corona is heated up to the temperature at least $20 \mathrm{MK}$. A flare on the back side of the Sun produces a flux of solar cosmic rays that observed at the Earth. The protons from the flare X8.2 came with a steep front and with the delay equal to the proton transit time along the line of the interplanetary magnetic field. The recorded protons could reach the Earth, propagating without collisions along the line of the interplanetary magnetic field of the Archimedes spiral. The results of observations of accelerated relativistic electrons on the SOHO/EPHIN instrument show that electrons are accelerated during flares and then propagated in the interplanetary space together with protons. A simple analysis of the GOES results with an estimation of the Larmor radii showed that the generation of cosmic rays does not reveal any correlation with the flux of relativistic electrons. At present, it is difficult to explain this contradiction. The discovery of flares appeared on dwarfs of class $G$ with release of the flare energy 3 to 4 orders of magnitude higher than by the flares on the Sun can be considered as a new argument in favor of the mechanism of galactic cosmic ray acceleration based on the current sheet rather than acceleration in shock waves. 


\section{Acknowledgments}

Authors are greatly thankful to the teams which obtained the data from GOES, SDO AIA, SDO HMI, SOHO MDI, and SOHO/EPHIN and represented them on the sites https://www.solarmonitor.org/goes_pop.php?date https://sdo.gsfc.nasa.gov/data/aiahmi/ https://www.solarmonitor.org/index.php?date http://www2.physik.uni-kiel.de/SOHO/phpeph/EPHIN.htm . We acknowledge Referee 2 for correction the text of paper.

\section{References}

Balabin, Yu.V, Vashenyuk, E.V., Mingalev, O.V., Podgorny, A.I., Podgorny I.M.: 2005, Astronomy Reports. 49, 837.

Istomin, Ya.N.: 2010, Mon. Not. R. Astron. Soc. 408, 1307.

Lin R.P., Krucker S., Hurford, G.J. et al.: 2003, Astrophys. J. 595, L69.

Maehara, H., Shibayama, T., Notsu, S., et al.: 2012, Nature. 485, 478.

Podgorny, A.I., Podgorny, I.M.: 2012, Geomagnetism and Aeronomy. 52, 162.

Podgorny, A.I., Podgorny, I.M.: 2013, Sun and Geosphere. 8, 71

Podgorny, I.M., Podgorny, A. I.: 2016, Sun and Geosphere. 11, 85.

Podgorny I.M., Podgorny A.I.: 2017, Proceedings of the 40th Annual Apatity Seminar (2-6 March, Apatity), p. 78.

Podgorny I.M., Podgorny A.I.: 2018, Sun and Geosphere. 13, 69.

Shibayama, T., Maehara, H., Notsu, S. et al.: 2013, Ap. J. Suplimento Ser. 209, 5. 